\title{
On the Area of the Fundamental Region of a Binary Form Associated with Algebraic Trigonometric Quantities
}

\author{
Anton Mosunov
}

\begin{abstract}
Let $F(x, y)$ be a binary form of degree at least three and non-zero discriminant. We estimate the area $A_{F}$ bounded by the curve $|F(x, y)|=1$ for four families of binary forms. The first two families that we are interested in are homogenizations of minimal polynomials of $2 \cos \left(\frac{2 \pi}{n}\right)$ and $2 \sin \left(\frac{2 \pi}{n}\right)$, which we denote by $\Psi_{n}(x, y)$ and $\Pi_{n}(x, y)$, respectively. The remaining two families of binary forms that we consider are homogenizations of Chebyshev polynomials of the first and second kinds, denoted $T_{n}(x, y)$ and $U_{n}(x, y)$, respectively.
\end{abstract}

\section{Introduction}

Let $F(x, y)$ be a binary form with real coefficients of degree $n \geq 3$ and non-zero discriminant $D_{F}$. The set

$$
\left\{(x, y) \in \mathbb{R}^{2}:|F(x, y)| \leq 1\right\},
$$

which represents the collection of all points bounded by the curve $|F(x, y)|=1$, is called the fundamental region of $F$. It was proved by Mahler [7] that the area $A_{F}$ of the fundamental region of $F$ is finite.

In what follows, we restrict our attention to binary forms $F$ having integer coefficients. The quantity $A_{F}$, which can be evaluated via the formula [1, Section 3]

$$
A_{F}=\int_{-\infty}^{+\infty} \frac{d x}{|F(x, 1)|^{2 / n}},
$$

plays a significant role in the analysis of certain Diophantine equations and inequalities associated with $F$. Mahler [7] proved that, for a positive integer $h$, the number of integer solutions $Z_{F}(h)$ to the Thue inequality $|F(x, y)| \leq h$ satisfies

$$
\left|Z_{F}(h)-A_{F} h^{2 / n}\right| \ll_{F} h^{1 /(n-1)} .
$$

In 2019, Stewart and Xiao 13 proved that the number of integers of absolute value at most $h$ which are represented by the form $F$ is asymptotic to $C_{F} h^{2 / n}$, 
where $C_{F}$ is a positive number which depends on $F$ and is a rational multiple of $A_{F}$.

The values $A_{F}$ for certain binary forms $F$ are intimately connected to the values of the beta function

$$
B(x, y)=\int_{0}^{1} t^{x-1}(1-t)^{y-1} d t
$$

where $x$ and $y$ are complex numbers with positive real parts. An overview of important properties of $B(x, y)$ is given in Section 2, For a matrix $M=\left(\begin{array}{ll}a & b \\ c & d\end{array}\right)$, with real coefficients, define

$$
F_{M}(x, y)=F(a x+b y, c x+d y) .
$$

Two forms $F$ and $G$ are said to be equivalent under $\mathrm{GL}_{2}(\mathbb{R})$ if $G=F_{M}$ for some $M \in \mathrm{GL}_{2}(\mathbb{R})$. Analogously, we define the equivalence of forms under $\mathrm{GL}_{2}(\mathbb{Z})$. In 1994, Bean [1, Corollary 1] proved that $A_{F}=3 B\left(\frac{1}{3}, \frac{1}{3}\right)=15.8997 \ldots$ when $F$ is a binary form that is equivalent under $\mathrm{GL}_{2}(\mathbb{Z})$ to $x y(x-y)$. Furthermore, he proved that this value is the largest among all binary forms with integer coefficients, degree at least three and non-zero discriminant.

It was proved by Stewart and Xiao [13, Corollary 1.3] that if $a, b$ are fixed non-zero integers and $F_{n}(x, y)=a x^{n}+b y^{n}$ is a binomial form of degree $n \geq 3$, then

$$
A_{F_{n}}= \begin{cases}\frac{1}{n|a b|^{1 / n}}\left(2 B\left(\frac{1}{n}, 1-\frac{2}{n}\right)+B\left(\frac{1}{n}, \frac{1}{n}\right)\right) & \text { if } n \text { is odd } \\ \frac{2}{n|a b|^{1 / n}} B\left(\frac{1}{n}, \frac{1}{n}\right) & \text { if } a b \text { is positive and } n \text { is even, } \\ \frac{4}{n|a b|^{1 / n}} B\left(\frac{1}{n}, 1-\frac{2}{n}\right) & \text { if } a b \text { is negative and } n \text { is even. }\end{cases}
$$

In Section 3 we prove that $\lim _{n \rightarrow \infty} A_{F_{n}}=4$.

In 2019, Fouvry and Waldschmidt [4, Théorème 1.5] estimated $A_{\Phi_{n}}$ for cyclotomic binary forms $\Phi_{n}$ (note that a positive integer $n$ no longer refers to the degree of the form). It is a consequence of their result that for any $\varepsilon>0$ there exists $n_{0}=n_{0}(\varepsilon)$ such that for all $n \geq n_{0}$ the inequalities

$$
\left(2-n^{-1+\varepsilon}\right)^{2}<A_{\Phi_{n}}<\left(2+n^{-1+\varepsilon}\right)^{2}
$$

are satisfied. Consequently, $\lim _{n \rightarrow \infty} A_{\Phi_{n}}=4$.

In this article we estimate $A_{F}$ for four families of binary forms. Let $\Psi_{n}(x)$ and $\Pi_{n}(x)$ denote the minimal polynomials of $2 \cos \left(\frac{2 \pi}{n}\right)$ and $2 \sin \left(\frac{2 \pi}{n}\right)$, respectively. The first two families that we are interested in are $\Psi_{n}(x, y)$ and $\Pi_{n}(x, y)$, which are homogenizations of $\Psi_{n}(x)$ and $\Pi_{n}(x)$, respectively. By [15, Lemma],

$$
\Psi_{n}(x, y)=\prod_{\substack{1 \leq k<\frac{n}{2} \\ \operatorname{gcd}(k, n)=1}}\left(x-2 \cos \left(\frac{2 \pi k}{n}\right) y\right) .
$$




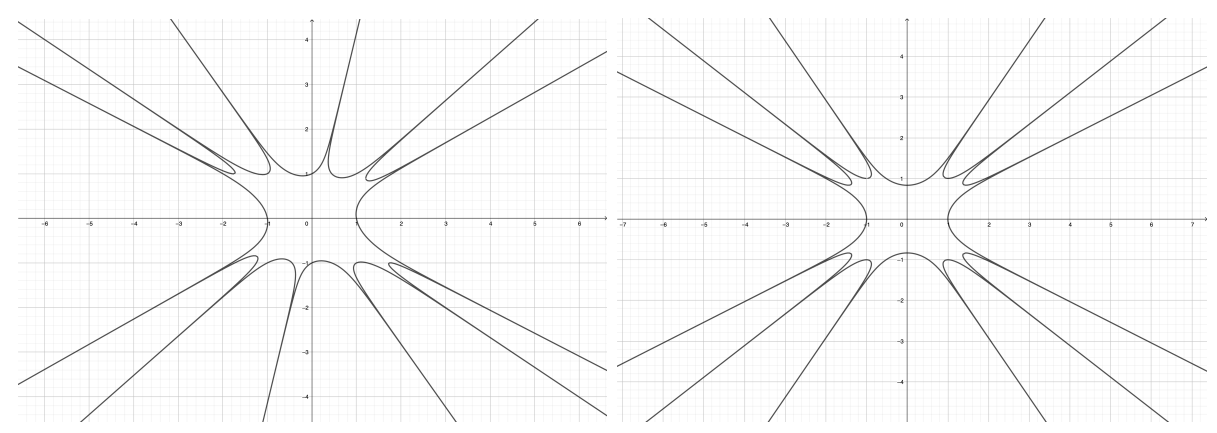

Figure 1: Graphs of $\left|\Psi_{13}(x, y)\right|=1$ (left) and $\left|\Pi_{9}(x, y)\right|=1$ (right)

Further, since $\sin \left(\frac{2 \pi}{n}\right)=\cos \left(\frac{2 \pi(n-4)}{4 n}\right)$, it follows from 4 that $\sin \left(\frac{2 \pi}{n}\right)$ is an algebraic conjugate of $\cos \left(\frac{2 \pi}{c(n)}\right)$, where $c(n)$ is the denominator of $\frac{n-4}{4 n}$ (in lowest terms). Consequently,

$$
\Pi_{n}(x, y)=\Psi_{c(n)}(x, y) .
$$

See Figure 1 for the graphs of $\left|\Psi_{13}(x, y)\right|=1$ and $\left|\Pi_{9}(x, y)\right|=1$. Note that in both cases the fundamental regions are not compact.

Next, let $T_{n}(x)$ and $U_{n}(x)$ denote Chebyshev polynomials of the first and second kinds, respectively. The other two families that we are interested in are $T_{n}(x, y)$ and $U_{n}(x, y)$, which are homogenizations of $T_{n}(x)$ and $U_{n}(x)$, respectively. It is known 8 ] that

$$
T_{n}(x, y)=2^{n-1} \prod_{k=0}^{n-1}\left(x-\cos \left(\frac{(2 k+1) \pi}{2 n}\right) y\right)
$$

and

$$
U_{n}(x, y)=2^{n} \prod_{k=1}^{n}\left(x-\cos \left(\frac{k \pi}{n+1}\right) y\right) .
$$

See Figure 2 for the graphs of $\left|T_{6}(x, y)\right|=1$ and $\left|U_{6}(x, y)\right|=1$. Note that in both cases the fundamental regions are not compact. It is also known that the binary forms $T_{n}(x, y)$ and $U_{n}(x, y)$ both have integer coefficients 8$]$.

For a positive integer $n$, let $d(n)$ denote the number of its positive divisors. Let $\varphi(n)$ denote the Euler's totient function. Our results are summarized in Theorem 1.1. Corollary 1.2, Theorem 1.3 and Theorem 1.4 . 


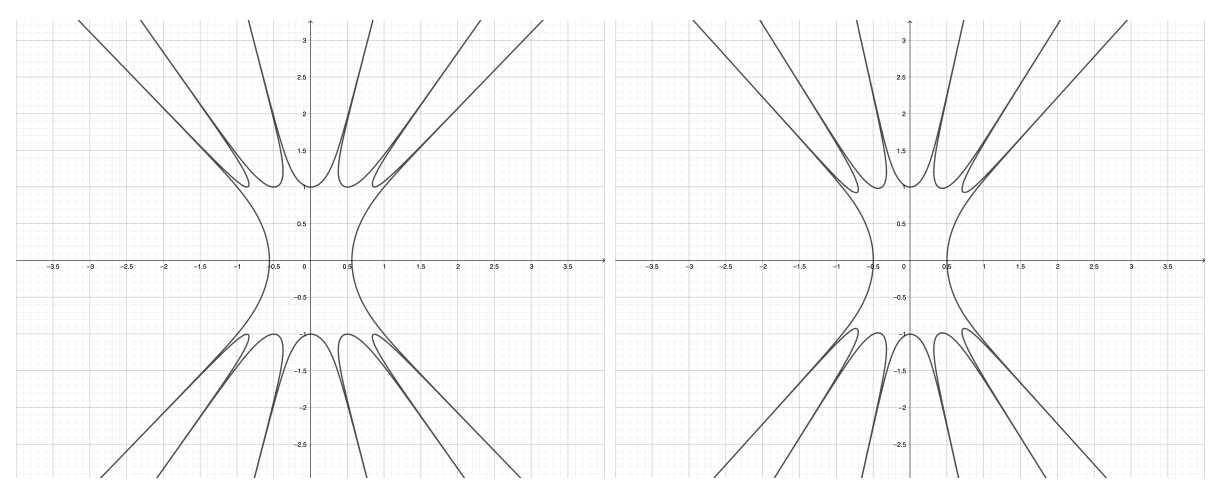

Figure 2: Graphs of $\left|T_{6}(x, y)\right|=1$ (left) and $\left|U_{6}(x, y)\right|=1$ (right)

Theorem 1.1. Let $n$ be a positive integer such that $\varphi(n) \geq 6$. Let $\Psi_{n}(x, y)$ denote the homogenization of the minimal polynomial of $2 \cos \left(\frac{2 \pi}{n}\right)$. Then

$$
\begin{aligned}
A_{\Psi_{n}} & >\frac{16}{3} \exp \left(-\frac{2 d(n) \log n}{\varphi(n)}\right) \\
A_{\Psi_{n}} & <2^{\frac{4}{\varphi(n)}} \exp \left(\frac{2 d(n)^{2} \log n}{\varphi(n)}\right) \times \\
& \times\left[\frac{16}{3}+2^{1-\frac{4}{\varphi(n)}} B\left(\frac{1}{2}, \frac{1}{2}-\frac{2}{\varphi(n)}\right)-2 \pi+\left(\frac{2}{n} B\left(\frac{1}{n}, 1-\frac{4}{\varphi(n)}\right)-2\right)-\left(\frac{2}{n} B\left(\frac{3}{n}, 1-\frac{4}{\varphi(n)}\right)-\frac{2}{3}\right)\right] .
\end{aligned}
$$

Furthermore, $\lim _{n \rightarrow \infty} A_{\Psi_{n}}=\frac{16}{3}$.

Corollary 1.2. Let $n$ be a positive integer. Let $\Pi_{n}(x, y)$ denote the homogenization of the minimal polynomial of $2 \sin \left(\frac{2 \pi}{n}\right)$. Then $\Pi_{n}(x, y)=\Psi_{c(n)}(x, y)$, where

$$
c(n)= \begin{cases}4 n & \text { if } n \text { is odd, } \\ 2 n & \text { if } n \equiv 2 \quad(\bmod 4), \\ n & \text { if } n \equiv 0 \quad(\bmod 8), \\ n / 2 & \text { if } n \equiv 12 \quad(\bmod 16), \\ n / 4 & \text { if } n \equiv 4 \quad(\bmod 16) .\end{cases}
$$

Furthermore, $\lim _{n \rightarrow \infty} A_{\Pi_{n}}=\frac{16}{3}$.

Theorem 1.3. Let $n$ be a positive integer such that $n \geq 3$. Let $T_{n}(x, y)$ denote the homogenization of the Chebyshev polynomial of the first kind $T_{n}(x)$. Then

$$
\frac{8}{3}<A_{T_{n}}<\frac{8}{3}+\frac{2}{3}(\sqrt[n]{4}-1)+B\left(\frac{1}{2}-\frac{1}{n}, \frac{1}{2}\right)-\pi
$$

Furthermore, $\lim _{n \rightarrow \infty} A_{T_{n}}=\frac{8}{3}$. 
Theorem 1.4. Let $n$ be a positive integer such that $n \geq 3$. Let $U_{n}(x, y)$ denote the homogenization of the Chebyshev polynomial of the second kind $U_{n}(x)$. Then

$$
\begin{aligned}
& A_{U_{n}}>\frac{8}{3}+\left(B\left(1+\frac{1}{n}, \frac{1}{2}\right)-2\right)+\frac{2}{3}\left((n+1)^{-2 / n}-1\right) \\
& A_{U_{n}}<\frac{8}{3}+\left(B\left(1+\frac{1}{n}, \frac{1}{2}\right)-2\right)+B\left(\frac{1}{2}-\frac{1}{n}, \frac{1}{2}\right)-\pi
\end{aligned}
$$

Furthermore, $\lim _{n \rightarrow \infty} A_{U_{n}}=\frac{8}{3}$.

Theorem 1.1 follows from Lemma 4.1, which we establish in Section 4 The proof of Corollary 1.2 is outlined in Section 5 . Theorem 1.3 follows from Lemma 6.1. which we establish in Section6. Theorem 1.4 follows from Lemma 7.1, which we establish in Section 7

Our last result, which we present in Section 8, concerns the quantity

$$
Q(F)=\left|D_{F}\right|^{1 / n(n-1)} A_{F}
$$

associated to a binary form $F$ of degree $n \geq 3$ and non-zero discriminant $D_{F}$. It was demonstrated by Bean [1] that the quantities $A_{F}$ and $Q(F)$ remain invariant for all binary forms that are equivalent under $\mathrm{GL}_{2}(\mathbb{Z})$ to $F$. Unlike $A_{F}$, the quantity $Q(F)$ also remains invariant for all binary forms that, up to multiplication by a complex number, are equivalent under $\mathrm{GL}_{2}(\mathbb{R})$ to $F$. An important conjecture about $Q(F)$ was formulated by Bean [2, Conjecture 1]. Let

$$
F_{n}^{*}(x, y)=\prod_{k=1}^{n}\left(\sin \left(\frac{k \pi}{n}\right) x-\cos \left(\frac{k \pi}{n}\right) y\right) .
$$

Conjecture 1.5. The maximum value $M_{n}$ of $Q(F)$ over the forms $F$ with complex coefficients of degree $n$ with discriminant $D_{F} \neq 0$ is attained precisely when $F$ is a form which, up to multiplication by a complex number, is equivalent under $\mathrm{GL}_{2}(\mathbb{R})$ to the form $F_{n}^{*}$. That is, $M_{n}=Q\left(F_{n}^{*}\right)$. Moreover, the limit of the sequence $\left\{M_{n}\right\}$ is $2 \pi$.

It was proved by Bean and Laugesen [3, Theorem 1] that

$$
Q\left(F_{n}^{*}\right)=2^{1-2 / n} n^{1 /(n-1)} B\left(\frac{1}{2}-\frac{1}{n}, \frac{1}{2}\right)
$$

and that $\lim _{n \rightarrow \infty} Q\left(F_{n}^{*}\right)=2 \pi$. Let $\nu_{2}(n)$ denote the 2 -adic order of $n$. In 9 it was proved by the author that the binary form

$$
S_{n}(x, y)=2^{n-1-\nu_{2}(n)} F_{n}^{*}(x, y)
$$

has integer coefficients and that the greatest common divisor of its coefficients is equal to one ${ }^{1}$ See Figure 3 for the graph of $\left|S_{6}(x, y)\right|=1$. Just like the graph

\footnotetext{
${ }^{1}$ Note that in [9, Proposition 1] where the coefficients of $F_{n}^{*}$ and $S_{n}$ are determined there should be no $Y$ in front of summation symbols.
} 


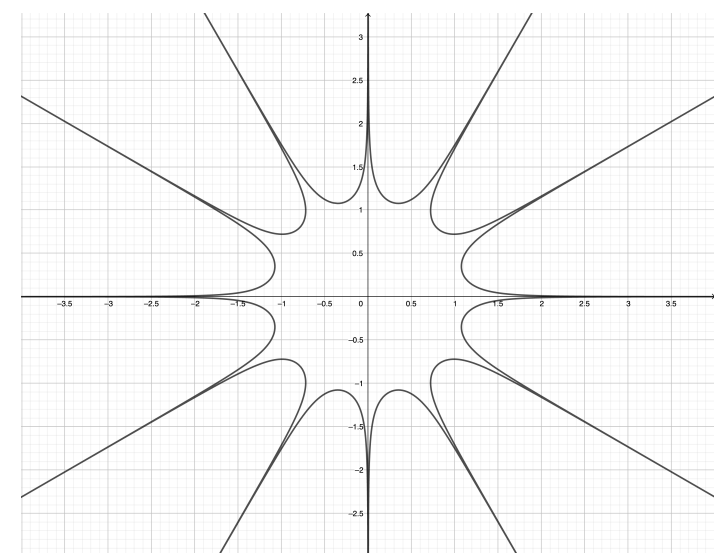

Figure 3: Graph of $\left|S_{6}(x, y)\right|=1$

of $\left|F_{n}^{*}(x, y)\right|=1$, the graph of $\left|S_{n}(x, y)\right|=1$ is invariant under rotation by any integer multiple of $\frac{\pi}{n}$. In [9] it was also demonstrated that

$$
A_{S_{n}}=4^{\nu_{2}(n) / n} B\left(\frac{1}{2}-\frac{1}{n}, \frac{1}{2}\right)
$$

and as a consequence that $\lim _{n \rightarrow \infty} A_{S_{n}}=\pi$. Since $Q\left(F_{n}^{*}\right)$ remains invariant under multiplication by a non-zero complex number, we see that $Q\left(S_{n}\right)=$ $Q\left(2^{n-1-\nu_{2}(n)} F_{n}^{*}\right)=Q\left(F_{n}^{*}\right)$. In Section 8 we will prove that $Q\left(T_{n}\right) \leq Q\left(S_{n}\right)$ and $Q\left(U_{n}\right) \leq Q\left(S_{n}\right)$ for every integer $n \geq 3$. We will also prove that

$$
\lim _{n \rightarrow \infty} Q\left(\Psi_{n}\right)=\lim _{n \rightarrow \infty} Q\left(\Pi_{n}\right)=\lim _{n \rightarrow \infty} Q\left(T_{n}\right)=\lim _{n \rightarrow \infty} Q\left(U_{n}\right)=\frac{16}{3} .
$$

\begin{tabular}{c|c|c|c|c|c}
$n$ & $Q\left(\Psi_{n}\right)$ & $Q\left(\Pi_{n}\right)$ & $Q\left(S_{n}\right)$ & $Q\left(T_{n}\right)$ & $Q\left(U_{n}\right)$ \\
\hline 3 & $\infty$ & $\infty$ & 15.8997 & 15.8997 & 15.8997 \\
4 & $\infty$ & $\infty$ & 11.7726 & 11.4798 & 11.5438 \\
5 & $\infty$ & 10.8953 & 10.3228 & 9.85622 & 9.94897 \\
6 & $\infty$ & $\infty$ & 9.55526 & 8.97702 & 9.08227 \\
7 & 15.8997 & 8.5577 & 9.06881 & 8.41412 & 8.52393 \\
8 & $\infty$ & $\infty$ & 8.72772 & 8.01814 & 8.12848 \\
9 & 15.8997 & 9.00056 & 8.47265 & 7.72218 & 7.83097
\end{tabular}

Table 1: Values of $Q\left(\Psi_{n}\right), Q\left(\Pi_{n}\right), Q\left(S_{n}\right), Q\left(T_{n}\right)$ and $Q\left(U_{n}\right)$ for $n=3,4, \ldots, 9$. 


\section{Beta Function and Its Properties}

For a complex number $z$ with positive real part, let

$$
\Gamma(z)=\int_{0}^{\infty} t^{z-1} e^{-t} d t
$$

denote the gamma function. This function satisfies the functional equation

$$
\Gamma(z+1)=z \Gamma(z) .
$$

The gamma function is analytic, with simple pole at $x=0$. Its residue at $x=0$ is equal to 1 , i.e.,

$$
\operatorname{Res}(\Gamma(x), 0)=\lim _{x \rightarrow 0} x \Gamma(x)=1 .
$$

The beta function (2) can be expressed in terms of the gamma function by means of the relation

$$
B(x, y)=\frac{\Gamma(x) \Gamma(y)}{\Gamma(x+y)} .
$$

If in 22 we make the change of variables $t=\sin ^{2} \theta$, then we obtain a trigonometric form of the beta function:

$$
B(x, y)=2 \int_{0}^{\pi / 2}(\sin \theta)^{2 x-1}(\cos \theta)^{2 y-1} d \theta .
$$

This representation of $B(x, y)$ is used in the derivation of the formula for $A_{F_{n}^{*}}$ 9. In Lemmas 4.1 6.1 and 7.1 we will use it to obtain upper and lower bounds on $A_{\Psi_{n}}, A_{T_{n}}$ and $A_{U_{n}}$.

Finally, we would like to remark that the values of beta function can be easily computed numerically, as this function is present in various mathematical software, such as Maple (Beta $(x, y))$, Mathematica $(\operatorname{Beta}(x, y))$ and MATLAB $(\operatorname{beta}(Z, W))$.

\section{The Limit of $A_{F_{n}}$ for $F_{n}(x, y)=a x^{n}+b y^{n}$}

In this section we prove the following result.

Proposition 3.1. Let $a, b$ and $n$ be non-zero integers with $n \geq 3$ and let $F_{n}(x, y)=a x^{n}+b y^{n}$. Then $\lim _{n \rightarrow \infty} A_{F_{n}}=4$.

Proof. In view of $\sqrt[3]{3}$ and $\lim _{n \rightarrow \infty}|a b|^{1 / n}=1$, the result will follow once we show that

$$
\lim _{n \rightarrow \infty} \frac{1}{n} B\left(\frac{1}{n}, 1-\frac{2}{n}\right)=1 \text { and } \lim _{n \rightarrow \infty} \frac{1}{n} B\left(\frac{1}{n}, \frac{1}{n}\right)=2 .
$$


To see that this is the case, first note that the identities

$$
B\left(\frac{1}{n}, 1-\frac{2}{n}\right)=\frac{\Gamma(1 / n) \Gamma(1-2 / n)}{\Gamma(1-1 / n)} \quad \text { and } \quad B\left(\frac{1}{n}, \frac{1}{n}\right)=\frac{\Gamma(1 / n)^{2}}{\Gamma(2 / n)}
$$

follow directly from $(12)$. It follows from (11) that

$$
\lim _{n \rightarrow \infty} \frac{\Gamma(1 / n)}{n}=1 \text { and } \lim _{n \rightarrow \infty} \frac{\Gamma(2 / n)}{n}=\frac{1}{2} .
$$

Since $\Gamma(1)=1$, we see that

$$
\lim _{n \rightarrow \infty} \frac{1}{n} B\left(\frac{1}{n}, 1-\frac{2}{n}\right)=\lim _{n \rightarrow \infty} \frac{\Gamma(1 / n) \Gamma(1-2 / n)}{n \Gamma(1-1 / n)}=\lim _{n \rightarrow \infty} \frac{\Gamma(1 / n)}{n}=1
$$

and

$$
\lim _{n \rightarrow \infty} \frac{1}{n} B\left(\frac{1}{n}, \frac{1}{n}\right)=\lim _{n \rightarrow \infty} \frac{\Gamma(1 / n)^{2}}{n \Gamma(2 / n)}=\frac{\lim _{n \rightarrow \infty}\left(\frac{\Gamma(1 / n)}{n}\right)^{2}}{\lim _{n \rightarrow \infty} \frac{\Gamma(2 / n)}{n}}=2 .
$$

\section{Upper and Lower Bounds on $A_{\Psi_{n}}$}

In this section we prove the following result.

Lemma 4.1. For any $n \in \mathbb{N}$ and $\alpha \in \mathbb{R}$ such that $\varphi(n) \geq 4$ and $2 / \varphi(n)<\alpha<1$,

$$
\begin{aligned}
\int_{-\infty}^{+\infty} \frac{d x}{\left|\Psi_{n}(x)\right|^{\alpha}} & >\left(4+\frac{16}{\varphi(n)^{2} \alpha^{2}-4}\right) \exp \left(-\frac{d(n) \log n}{2} \alpha\right) \\
\int_{-\infty}^{+\infty} \frac{d x}{\left|\Psi_{n}(x)\right|^{\alpha}} & <2^{\alpha} \exp \left(\frac{d(n)^{2} \log n}{2} \alpha\right), \times \\
\times & {\left[4+2^{1-\alpha} B\left(\frac{1-\alpha}{2}, \frac{1}{2}\right)-2 \pi+\frac{2}{n} B\left(\frac{\varphi(n) \alpha-2}{2 n}, 1-\alpha\right)-\frac{2}{n} B\left(\frac{\varphi(n) \alpha+2}{2 n}, 1-\alpha\right)\right] . }
\end{aligned}
$$

Let us see why Theorem 1.1 directly follows from Lemma 4.1 .

Proof of Theorem 1.1. It follows from (4) that the degree of $\Psi_{n}$ is equal to $\varphi(n) / 2$. Since the area $A_{\Psi_{n}}$ bounded by the curve $\left|\Psi_{n}(x, y)\right|=1$ can be computed via (1), the inequalities (5) follow from Lemma 4.1 once we take $\alpha=4 / \varphi(n)$.

The fact that $\lim _{n \rightarrow \infty} A_{\Psi_{n}}=\frac{16}{3}$ is a consequence of the following:

$$
\lim _{n \rightarrow \infty} B\left(\frac{1}{2}, \frac{1}{2}-\frac{2}{\varphi(n)}\right)=B\left(\frac{1}{2}, \frac{1}{2}\right)=\frac{\Gamma(1 / 2)^{2}}{\Gamma(1)}=\pi,
$$




$$
\begin{aligned}
& \lim _{n \rightarrow \infty} \frac{2}{n} B\left(\frac{1}{n}, 1-\frac{4}{\varphi(n)}\right)=\lim _{n \rightarrow \infty} \frac{2}{n} \Gamma\left(\frac{1}{n}\right)=2, \\
& \lim _{n \rightarrow \infty} \frac{2}{n} B\left(\frac{3}{n}, 1-\frac{4}{\varphi(n)}\right)=\lim _{n \rightarrow \infty} \frac{2}{n} \Gamma\left(\frac{3}{n}\right)=\frac{2}{3} .
\end{aligned}
$$

Further, we have

$$
\lim _{n \rightarrow \infty} \frac{2 d(n) \log n}{\varphi(n)}=0 \quad \text { and } \quad \lim _{n \rightarrow \infty} \frac{2 d(n)^{2} \log n}{\varphi(n)}=0 .
$$

These two limits follow from the fact that, for every integer $n>2$,

$$
d(n) \leq n^{\frac{1.067}{\log \log n}}
$$

and

$$
\varphi(n)>\frac{n}{e^{\gamma} \log \log n+\frac{3}{\log \log n}} .
$$

Here $\gamma \approx 0.5772$ denotes the Euler-Mascheroni constant. The upper bound on $d(n)$ was derived by Nicolas and Robin [10, while the lower bound on $\varphi(n)$ can be found in the paper of Rosser and Schoenfeld [12, Theorem 15].

Before proving Lemma 4.1, we need to establish three lemmas.

Lemma 4.2. Let $n$ be an integer such that $n \geq 3$. Then, for every real number $\theta$,

$$
\begin{aligned}
& \Psi_{n}( \pm 2 \cos \theta)=( \pm 1)^{\frac{\varphi(n)}{2}} e^{-\frac{\varphi(n)}{2} \theta i} \Phi_{n}\left( \pm e^{\theta i}\right), \\
& \Psi_{n}( \pm 2 \cosh \theta)=( \pm 1)^{\frac{\varphi(n)}{2}} e^{\frac{\varphi(n)}{2} \theta} \Phi_{n}\left( \pm e^{-\theta}\right) .
\end{aligned}
$$

Proof. According to Lehmer [5],

$$
\Psi_{n}\left(x+x^{-1}\right)=x^{-\varphi(n) / 2} \Phi_{n}(x) .
$$

Putting $x=e^{\theta i}$, we obtain the identity $(16)$, where the plus signs are chosen everywhere. Replacing $\theta$ with $\pi+\theta, \theta i$ and $\pi+\theta i$ we obtain the identity (16) where the minus signs are chosen everywhere, as well as the two identities in (17).

For a polynomial $f(x)$, let $L(f)$ denote the sum of the absolute values of coefficients of $f(x)$. The following lemma can be found in [4, Lemme 4.1].

Lemma 4.3. For every integer $n>1, L\left(\Phi_{n}\right) \leq n^{\frac{d(n)}{2}}$.

Lemma 4.4. For every positive integer $n$,

$$
\prod_{\substack{m \mid n \\ m \neq 1}} L\left(\Phi_{m}\right) \leq \exp \left(\frac{d(n)^{2} \log n}{2}\right) .
$$


Proof. By Lemma 4.3 .

$$
\begin{aligned}
\prod_{\substack{m \mid n \\
m \neq 1}} L\left(\Phi_{m}\right) & \leq \prod_{m \mid n} m^{\frac{d(m)}{2}} \\
& =\exp \left(\frac{1}{2} \sum_{m \mid n} d(m) \log m\right) \\
& \leq \exp \left(\frac{d(n) \log n}{2} \sum_{m \mid n} 1\right) \\
& =\exp \left(\frac{d(n)^{2} \log n}{2}\right) .
\end{aligned}
$$

Proof of Lemma 4.1. Note that

$$
\begin{aligned}
\int_{-\infty}^{+\infty} \frac{d x}{\left|\Psi_{n}(x)\right|^{\alpha}} & =\int_{-2}^{2} \frac{d x}{\left|\Psi_{n}(x)\right|^{\alpha}}+\int_{2}^{+\infty} \frac{d x}{\left|\Psi_{n}(x)\right|^{\alpha}}+\int_{-\infty}^{-2} \frac{d x}{\left|\Psi_{n}(x)\right|^{\alpha}} \\
& =-\int_{0}^{\pi} \frac{d(2 \cos \theta)}{\left|\Psi_{n}(2 \cos \theta)\right|^{\alpha}}+\int_{0}^{+\infty} \frac{d(2 \cosh \theta)}{\left|\Psi_{n}(2 \cosh \theta)\right|^{\alpha}}+\int_{-\infty}^{0} \frac{d(-2 \cosh \theta)}{\left|\Psi_{n}(-2 \cosh \theta)\right|^{\alpha}} \\
& =2 \int_{0}^{\pi} \frac{\sin \theta d \theta}{\left|\Psi_{n}(2 \cos \theta)\right|^{\alpha}}+2 \int_{0}^{+\infty} \frac{\sinh \theta d \theta}{\left|\Psi_{n}(2 \cosh \theta)\right|^{\alpha}}+2 \int_{0}^{+\infty} \frac{\sinh \theta d \theta}{\left|\Psi_{n}(-2 \cosh \theta)\right|^{\alpha}} \\
& =2 \int_{0}^{\pi} \frac{\sin \theta d \theta}{\left|\Phi_{n}\left(e^{\theta i}\right)\right|^{\alpha}}+2 \int_{0}^{+\infty} \frac{\sinh \theta d \theta}{e^{(\varphi(n) / 2) \alpha \theta} \Phi_{n}\left(e^{-\theta}\right)^{\alpha}}+2 \int_{0}^{+\infty} \frac{\sinh \theta d \theta}{e^{(\varphi(n) / 2) \alpha \theta} \Phi_{n}\left(-e^{-\theta}\right)^{\alpha}}
\end{aligned}
$$

where the last equality follows from Lemma 4.2. Let

$$
C_{1}=2 \int_{0}^{\pi} \frac{\sin \theta d \theta}{\left|\Phi_{n}\left(e^{\theta i}\right)\right|^{\alpha}}, \quad C_{2}=2 \int_{0}^{+\infty} \frac{\sinh \theta d \theta}{e^{(\varphi(n) / 2) \alpha \theta} \Phi_{n}\left(e^{-\theta}\right)^{\alpha}}, \quad C_{3}=2 \int_{0}^{+\infty} \frac{\sinh \theta d \theta}{e^{(\varphi(n) / 2) \alpha \theta} \Phi_{n}\left(-e^{-\theta}\right)^{\alpha}} .
$$

We will now derive upper and lower bounds on $C_{1}, C_{2}$ and $C_{3}$.

We begin with the derivation of a lower bound. By Lemma 4.3 ,

$$
\left|\Phi_{n}(z)\right| \leq L\left(\Phi_{n}\right) \max \{1,|z|\}^{\varphi(n)} \leq n^{\frac{d(n)}{2}}
$$


for all $z \in \mathbb{C}$ such that $|z| \leq 1$. In view of this,

$$
\begin{aligned}
C_{1} & =2 \int_{0}^{\pi} \frac{\sin \theta d \theta}{\left|\Phi_{n}\left(e^{\theta i}\right)\right|^{\alpha}} \\
& \geq 2 n^{-\frac{d(n)}{2} \alpha} \int_{0}^{\pi} \sin \theta d \theta \\
& =4 \exp \left(-\frac{d(n) \log n}{2} \alpha\right)
\end{aligned}
$$

and

$$
\begin{aligned}
C_{2} & =2 \int_{0}^{+\infty} \frac{\sinh \theta d \theta}{e^{(\varphi(n) / 2) \alpha \theta} \Phi_{n}\left(e^{-\theta}\right)^{\alpha}} \\
& \geq 2 n^{-\frac{d(n)}{2} \alpha} \int_{0}^{+\infty} e^{-\frac{\varphi(n)}{2} \alpha \theta} \sinh \theta d \theta \\
& =2 n^{-\frac{d(n)}{2} \alpha} \frac{1}{\left(\frac{\varphi(n)}{2} \alpha\right)^{2}-1} \\
& =\frac{8}{\varphi(n)^{2} \alpha^{2}-4} \exp \left(-\frac{d(n) \log n}{2} \alpha\right) .
\end{aligned}
$$

Similarly,

$$
C_{3} \geq \frac{8}{\varphi(n)^{2} \alpha^{2}-4} \exp \left(-\frac{d(n) \log n}{2} \alpha\right) .
$$

We conclude that

$$
\int_{-\infty}^{+\infty} \frac{d x}{\left|\Psi_{n}(x)\right|^{\alpha}}=C_{1}+C_{2}+C_{3} \geq\left(4+\frac{16}{\varphi(n)^{2} \alpha^{2}-4}\right) \exp \left(-\frac{d(n) \log n}{2} \alpha\right) .
$$

To derive an upper bound, we will make use of the identity

$$
z^{n}-1=\prod_{m \mid n} \Phi_{m}(z) .
$$

Notice that

$$
\Phi_{n}(z)=\left(z^{n}-1\right) \prod_{\substack{m \mid n \\ m \neq n}} \Phi_{m}(z)^{-1}
$$


Thus for every $z \in \mathbb{C}$ such that $|z|<1$ we have

$$
\begin{aligned}
\left|\Phi_{n}(z)\right|^{-1} & =\frac{1}{\left|z^{n}-1\right|} \prod_{\substack{m \mid n \\
m \neq n}}\left|\Phi_{m}(z)\right| \\
& =\frac{|z-1|}{\left|z^{n}-1\right|} \prod_{\substack{m \mid n \\
1<m<n}}\left|\Phi_{m}(z)\right| \\
& \leq \frac{|z-1|}{\left|z^{n}-1\right|} \prod_{\substack{m \mid n \\
m \neq 1}} L\left(\Phi_{m}\right) \\
& <\frac{2}{\left|z^{n}-1\right|} \exp \left(\frac{d(n)^{2} \log n}{2}\right),
\end{aligned}
$$

where the last inequality follows from Lemma 4.4. In view of this,

$$
\begin{aligned}
C_{1} & =2 \int_{0}^{\pi} \frac{\sin \theta d \theta}{\left|\Phi_{n}\left(e^{\theta i}\right)\right|^{\alpha}} \\
& \leq 2^{1+\alpha} \exp \left(\frac{d(n)^{2} \log n}{2} \alpha\right) \int_{0}^{\pi} \frac{\sin \theta d \theta}{\left|e^{n \theta i}-1\right|^{\alpha}} \\
& =2^{1+\alpha} \exp \left(\frac{d(n)^{2} \log n}{2} \alpha\right)\left(\int_{0}^{\pi} \sin \theta d \theta+\int_{0}^{\pi} \sin \theta\left(\left|e^{n \theta i}-1\right|^{-\alpha}-1\right) d \theta\right) \\
& \leq 2^{1+\alpha} \exp \left(\frac{d(n)^{2} \log n}{2} \alpha\right)\left(2+\int_{0}^{\pi}\left(\frac{1}{\left|e^{n \theta i}-1\right|^{\alpha}}-1\right) d \theta\right) \\
& =2^{1+\alpha} \exp \left(\frac{d(n)^{2} \log n}{2} \alpha\right)\left(2+\int_{0}^{\pi} \frac{d \theta}{\left|e^{n \theta i}-1\right|^{\alpha}}-\pi\right) .
\end{aligned}
$$

It remains to estimate the integral $\int_{0}^{\pi} \frac{d \theta}{\left|e^{n \theta i}-1\right|^{\alpha}}$. Note that

$$
\left|e^{n \theta i}-1\right|^{2}=(\cos (n \theta)-1)^{2}+\sin ^{2}(n \theta)=2-2 \cos (n \theta)=4 \sin ^{2}\left(\frac{n \theta}{2}\right) .
$$


Therefore,

$$
\begin{aligned}
\int_{0}^{\pi} \frac{d \theta}{\left|e^{n \theta i}-1\right|^{\alpha}} & =2^{-\alpha} \int_{0}^{\pi}\left|\sin \left(\frac{n \theta}{2}\right)\right|^{-\alpha} d \theta \\
& =\frac{2^{1-\alpha}}{n} \int_{0}^{n \pi / 2}|\sin \theta|^{-\alpha} d \theta \\
& =2^{-\alpha} \cdot 2 \int_{0}^{\pi / 2}(\sin \theta)^{-\alpha} d \theta \\
& =2^{-\alpha} B\left(\frac{1-\alpha}{2}, \frac{1}{2}\right)
\end{aligned}
$$

We conclude that

$$
\begin{aligned}
C_{1} & =2 \int_{0}^{\pi} \frac{\sin \theta d \theta}{\left|\Phi_{n}\left(e^{\theta i}\right)\right|^{\alpha}} \\
& \leq 2^{\alpha} \exp \left(\frac{d(n)^{2} \log n}{2} \alpha\right)\left(4+2^{1-\alpha} B\left(\frac{1-\alpha}{2}, \frac{1}{2}\right)-2 \pi\right) .
\end{aligned}
$$

Next, we derive an upper bound on $C_{2}$ :

$$
\begin{aligned}
C_{2} & =2 \int_{0}^{+\infty} \frac{\sinh \theta d \theta}{e^{(\varphi(n) / 2) \alpha \theta} \Phi_{n}\left(e^{-\theta}\right)^{\alpha}} \\
& \leq 2^{1+\alpha} \exp \left(\frac{d(n)^{2} \log n}{2} \alpha\right) \int_{0}^{+\infty} \frac{\sinh \theta d \theta}{e^{(\varphi(n) / 2) \alpha \theta}\left(1-e^{-n \theta}\right)^{\alpha}} \\
& =2^{1+\alpha} \exp \left(\frac{d(n)^{2} \log n}{2} \alpha\right) \frac{1}{2 n} \int_{0}^{1}\left(t^{\frac{\varphi(n) \alpha-2}{2 n}-1}-t^{\frac{\varphi(n) \alpha+2}{2 n}-1}\right)(1-t)^{-\alpha} d t \\
& =2^{\alpha} \exp \left(\frac{d(n)^{2} \log n}{2} \alpha\right)\left(\frac{1}{n} B\left(\frac{\varphi(n) \alpha-2}{2 n}, 1-\alpha\right)-\frac{1}{n} B\left(\frac{\varphi(n) \alpha+2}{2 n}, 1-\alpha\right)\right),
\end{aligned}
$$

where the second-to-last equality follows from the change of variables $t=e^{-n \theta}$.

Finally, we derive an upper bound on $C_{3}$. We consider two cases. 
Case 1. Suppose that $n$ is even. Then

$$
\begin{aligned}
C_{3} & =2 \int_{0}^{+\infty} \frac{\sinh \theta d \theta}{e^{(\varphi(n) / 2) \alpha \theta} \Phi_{n}\left(-e^{-\theta}\right)^{\alpha}} \\
& \leq 2^{1+\alpha} \exp \left(\frac{d(n)^{2} \log n}{2} \alpha\right) \int_{0}^{+\infty} \frac{\sinh \theta d \theta}{e^{(\varphi(n) / 2) \alpha \theta}\left(1-\left(-e^{-\theta}\right)^{n}\right)^{\alpha}} \\
& =2^{1+\alpha} \exp \left(\frac{d(n)^{2} \log n}{2} \alpha\right) \int_{0}^{+\infty} \frac{\sinh \theta d \theta}{e^{(\varphi(n) / 2) \alpha \theta}\left(1-e^{-n \theta}\right)^{\alpha}} \\
& =2^{\alpha} \exp \left(\frac{d(n)^{2} \log n}{2} \alpha\right)\left(\frac{1}{n} B\left(\frac{\varphi(n) \alpha-2}{2 n}, 1-\alpha\right)-\frac{1}{n} B\left(\frac{\varphi(n) \alpha+2}{2 n}, 1-\alpha\right)\right),
\end{aligned}
$$

where the last equality was derived from the estimate for $C_{2}$.

Case 2. Suppose that $n$ is odd. Then for any real number $x$ we have $\Phi_{n}(-x)=\Phi_{2 n}(x)$, so

$$
\begin{aligned}
C_{3} & =2 \int_{0}^{+\infty} \frac{\sinh \theta d \theta}{e^{(\varphi(n) / 2) \alpha \theta} \Phi_{n}\left(-e^{-\theta}\right)^{\alpha}} \\
& =2 \int_{0}^{+\infty} \frac{\sinh \theta d \theta}{e^{(\varphi(n) / 2) \alpha \theta} \Phi_{2 n}\left(e^{-\theta}\right)^{\alpha}} \\
& \leq 2^{1+\alpha} \exp \left(\frac{d(n)^{2} \log n}{2} \alpha\right) \int_{0}^{+\infty} \frac{\sinh \theta d \theta}{e^{(\varphi(n) / 2) \alpha \theta}\left(1-e^{-2 n \theta}\right)^{\alpha}} \\
& \leq 2^{1+\alpha} \exp \left(\frac{d(n)^{2} \log n}{2} \alpha\right) \int_{0}^{+\infty} \frac{\sinh \theta d \theta}{e^{(\varphi(n) / 2) \alpha \theta}\left(1-e^{-n \theta}\right)^{\alpha}} \\
& =2^{\alpha} \exp \left(\frac{d(n)^{2} \log n}{2} \alpha\right)\left(\frac{1}{n} B\left(\frac{\varphi(n) \alpha-2}{2 n}, 1-\alpha\right)-\frac{1}{n} B\left(\frac{\varphi(n) \alpha+2}{2 n}, 1-\alpha\right)\right)
\end{aligned}
$$

where the last equality was derived from the estimate for $C_{2}$.

We conclude that

$$
\begin{aligned}
\int_{-\infty}^{+\infty} \frac{d x}{\left|\Psi_{n}(x)\right|^{\alpha}} & =C_{1}+C_{2}+C_{3} \\
& \leq 2^{\alpha} \exp \left(\frac{d(n)^{2} \log n}{2} \alpha\right) \times \\
& \times\left(4+2^{1-\alpha} B\left(\frac{1-\alpha}{2}, \frac{1}{2}\right)-2 \pi+\frac{2}{n} B\left(\frac{\varphi(n) \alpha-2}{2 n}, 1-\alpha\right)-\frac{2}{n} B\left(\frac{\varphi(n) \alpha+2}{2 n}, 1-\alpha\right)\right) .
\end{aligned}
$$




\section{Proof of Corollary 1.2}

In view of the identity $\sin \left(\frac{2 \pi}{n}\right)=\cos \left(\frac{2 \pi(n-4)}{4 n}\right)$, we can follow the argument of Niven [11, III.4] and consider the following five cases.

(1) If $n$ is odd, then the fraction $\frac{n-4}{4 n}$ is in lowest terms. Thus $2 \cos \left(\frac{2 \pi(n-4)}{4 n}\right)$ is conjugate to $2 \cos \left(\frac{2 \pi}{4 n}\right)$ and $c(n)=4 n$.

(2) If $n \equiv 2(\bmod 4)$, then $\frac{n-4}{4 n}$ reduces to a fraction with denominator $2 n$. Thus $2 \cos \left(\frac{2 \pi(n-4)}{4 n}\right)$ is conjugate to $2 \cos \left(\frac{2 \pi}{2 n}\right)$ and $c(n)=2 n$.

(3) If $n \equiv 0(\bmod 8)$, then $\frac{n-4}{4 n}$ reduces to a fraction with denominator $n$. Thus $2 \cos \left(\frac{2 \pi(n-4)}{4 n}\right)$ is conjugate to $2 \cos \left(\frac{2 \pi}{n}\right)$ and $c(n)=n$.

(4) If $n \equiv 12(\bmod 16)$, then $\frac{n-4}{4 n}$ reduces to a fraction with denominator $n / 2$. Thus $2 \cos \left(\frac{2 \pi(n-4)}{4 n}\right)$ is conjugate to $2 \cos \left(\frac{2 \pi}{n / 2}\right)$ and $c(n)=n / 2$.

(5) If $n \equiv 4(\bmod 16)$, then $\frac{n-4}{4 n}$ reduces to a fraction with denominator $n / 4$. Thus $2 \cos \left(\frac{2 \pi(n-4)}{4 n}\right)$ is conjugate to $2 \cos \left(\frac{2 \pi}{n / 4}\right)$ and $c(n)=n / 4$.

A combination of this result with Theorem 1.1 yields $\lim _{n \rightarrow \infty} A_{\Pi_{n}}=\lim _{n \rightarrow \infty} A_{\Psi_{n}}=\frac{16}{3}$.

\section{Upper and Lower Bounds on $A_{T_{n}}$}

In this section we prove the following result.

Lemma 6.1. For any $n \in \mathbb{N}$ and $\alpha \in \mathbb{R}$ such that $n \geq 3$ and $2 / n \leq \alpha<1$,

$$
2+\frac{2}{n^{2} \alpha^{2}-1}<\int_{-\infty}^{+\infty} \frac{d x}{\left|T_{n}(x)\right|^{\alpha}}<2+\frac{2^{1+\alpha}}{n^{2} \alpha^{2}-1}+B\left(\frac{1-\alpha}{2}, \frac{1}{2}\right)-\pi .
$$

Let us see why Theorem 1.3 directly follows from Lemma 6.1.

Proof of Theorem 1.3. Since for an arbitrary binary form $F$ of positive degree $n$ the area bounded by the curve $|F(x, y)|=1$ can be computed via (1), the inequalities (6) follow from Lemma 6.1 if we take $\alpha=2 / n$.

To see that $\lim _{n \rightarrow \infty} A_{T_{n}}=\frac{8}{3}$, it is sufficient to demonstrate that the limit of $B\left(\frac{1}{2}-\frac{1}{n}, \frac{1}{2}\right)$ as $n$ approaches infinity is equal to $\pi$. Recall that the gamma function (9) satisfies $\Gamma(1)=1$ and $\Gamma(1 / 2)=\sqrt{\pi}$. In view of $(12)$, we see that

$$
\lim _{n \rightarrow \infty} B\left(\frac{1}{2}-\frac{1}{n}, \frac{1}{2}\right)=B\left(\frac{1}{2}, \frac{1}{2}\right)=\frac{\Gamma(1 / 2)^{2}}{\Gamma(1)}=\pi .
$$


We will now turn our attention to the proof of Lemma 6.1.

Proof of Lemma 6.1. Since the function $\left|T_{n}(x)\right|$ is even, we find that

$$
\int_{-\infty}^{+\infty} \frac{d x}{\left|T_{n}(x)\right|^{\alpha}}=C_{1}+C_{2}
$$

where

$$
C_{1}=2 \int_{1}^{+\infty} \frac{d x}{T_{n}(x)^{\alpha}}, \quad C_{2}=2 \int_{0}^{1} \frac{d x}{\left|T_{n}(x)\right|^{\alpha}},
$$

We will now derive upper and lower bounds on $C_{1}$ and $C_{2}$.

First, we determine upper and lower bounds on $C_{1}$. Recall that

$$
T_{n}(x)=\frac{\left(x+\sqrt{x^{2}-1}\right)^{n}+\left(x-\sqrt{x^{2}-1}\right)^{n}}{2}
$$

for all $x$ such that $|x| \geq 1$. Hence

$$
\frac{\left(x+\sqrt{x^{2}-1}\right)^{n}}{2}<T_{n}(x)<\left(x+\sqrt{x^{2}-1}\right)^{n}
$$

for all $x>1$. Using the lower bound in (18), as well as the change of variables $x=\csc \theta$, we find that

$$
\begin{aligned}
C_{1} & =2 \int_{1}^{+\infty} \frac{d x}{T_{n}(x)^{\alpha}} \\
& <2^{\alpha+1} \int_{1}^{+\infty} \frac{d x}{\left(x+\sqrt{x^{2}-1}\right)^{n \alpha}} \\
& =2^{\alpha+1} \int_{0}^{\pi / 2} \frac{(\cos \theta)(\sin \theta)^{n \alpha-2} d \theta}{(1+\cos \theta)^{n \alpha}} \\
& =\left.2^{\alpha+1} \frac{(\sin \theta)^{n \alpha-1}(n \alpha \cos \theta+1)}{\left(n^{2} \alpha^{2}-1\right)(1+\cos \theta)^{n \alpha}}\right|_{0} ^{\pi / 2} \\
& =\frac{2^{\alpha+1}}{n^{2} \alpha^{2}-1}
\end{aligned}
$$

A lower bound on $C_{1}$ can be obtained analogously. As a result, we get

$$
\frac{2}{n^{2} \alpha^{2}-1}<C_{1}<\frac{2^{1+\alpha}}{n^{2} \alpha^{2}-1} .
$$

Next, we determine upper and lower bounds on $C_{2}$. We apply the substitution $x=\cos (\theta / n)$ and make use of the identity $\cos \theta=T_{n}(\cos (\theta / n))$ as 
follows:

$$
\begin{aligned}
C_{2} & =2 \int_{0}^{1} \frac{d x}{\left|T_{n}(x)\right|^{\alpha}} \\
& =\frac{2}{n} \int_{0}^{\pi n / 2} \frac{\sin (\theta / n) d \theta}{|\cos \theta|^{\alpha}} \\
& =\frac{2}{n} \int_{0}^{\pi n / 2} \sin (\theta / n) d \theta+\varepsilon_{n},
\end{aligned}
$$

where

$$
\varepsilon_{n}=\frac{2}{n} \int_{0}^{\pi n / 2} \sin (\theta / n)\left(|\cos \theta|^{-\alpha}-1\right) d \theta .
$$

Note that $\varepsilon_{n}>0$. Since

$$
\frac{2}{n} \int_{0}^{\pi n / 2} \sin (\theta / n) d \theta=2 \int_{0}^{\pi / 2} \sin \theta d \theta=2,
$$

we have

$$
C_{2}=\frac{2}{n} \int_{0}^{\pi n / 2} \sin (\theta / n) d \theta+\varepsilon_{n}>2 .
$$

It remains to find an upper bound on $C_{2}$. Note that

$$
\begin{aligned}
\varepsilon_{n} & =\frac{2}{n} \int_{0}^{\pi n / 2} \sin (\theta / n)\left(|\cos \theta|^{-\alpha}-1\right) d \theta \\
& \leq \frac{2}{n} \int_{0}^{\pi n / 2}|\cos \theta|^{-\alpha} d \theta-\pi \\
& =2 \int_{0}^{\pi / 2}(\cos \theta)^{-\alpha} d \theta-\pi \\
& =B\left(\frac{1-\alpha}{2}, \frac{1}{2}\right)-\pi,
\end{aligned}
$$

where the last equality follows from 13 . We conclude that

$$
2<C_{2} \leq 2+B\left(\frac{1-\alpha}{2}, \frac{1}{2}\right)-\pi
$$

\footnotetext{
${ }^{2}$ The author is grateful to Fedor Petrov for recognizing the third equality. See https: //mathoverflow.net/questions/345820/an-integral-of-sinx-cosnx-2-n-from-pi-to-pi
} 


\section{Upper and Lower Bounds on $A_{U_{n}}$}

In this section we prove the following result.

Lemma 7.1. For any $n \in \mathbb{N}$ and $\alpha \in \mathbb{R}$ such that $n \geq 3$ and $2 / n \leq \alpha<1$,

$$
\begin{aligned}
& \int_{-\infty}^{+\infty} \frac{d x}{\left|U_{n}(x)\right|^{\alpha}}>B\left(\frac{2+\alpha}{2}, \frac{1}{2}\right)+\frac{2}{n^{2} \alpha^{2}-1}(n+1)^{-\alpha} \\
& \int_{-\infty}^{+\infty} \frac{d x}{\left|U_{n}(x)\right|^{\alpha}}<B\left(\frac{2+\alpha}{2}, \frac{1}{2}\right)+\frac{2}{n^{2} \alpha^{2}-1}+B\left(\frac{1-\alpha}{2}, \frac{1}{2}\right)-\pi
\end{aligned}
$$

Let us see why Theorem 1.4 directly follows from Lemma 7.1 .

Proof of Theorem 1.4. Since for an arbitrary binary form $F$ of positive degree $n$ the area bounded by the curve $|F(x, y)|=1$ can be computed via (1), the inequalities (7) follow from Lemma 7.1 if we take $\alpha=2 / n$.

To see that $\lim _{n \rightarrow \infty} A_{U_{n}}=\frac{8}{3}$, it is sufficient to demonstrate that the limits of $B\left(\frac{1}{2}-\frac{1}{n}, \frac{1}{2}\right)$ and $B\left(1+\frac{1}{n}, \frac{1}{2}\right)$ as $n$ approaches infinity are equal to $\pi$ and 2 , respectively. Recall that the gamma function (9) satisfies $\Gamma(1)=1$ and $\Gamma(1 / 2)=\sqrt{\pi}$. In view of $\sqrt{12}$, we see that

$$
\lim _{n \rightarrow \infty} B\left(\frac{1}{2}-\frac{1}{n}, \frac{1}{2}\right)=B\left(\frac{1}{2}, \frac{1}{2}\right)=\frac{\Gamma(1 / 2)^{2}}{\Gamma(1)}=\pi
$$

and

$$
\lim _{n \rightarrow \infty} B\left(1+\frac{1}{n}, \frac{1}{2}\right)=B\left(1, \frac{1}{2}\right)=\frac{\Gamma(1) \Gamma(1 / 2)}{\Gamma(3 / 2)}=\frac{\Gamma(1) \Gamma(1 / 2)}{(1 / 2) \Gamma(1 / 2)}=2,
$$

where the second-to-last equality follows from the functional equation 10 .

We will now turn our attention to the proof of Lemma 7.1.

Proof of Lemma 7.1. Since the function $\left|U_{n}(x)\right|$ is even, we find that

$$
\int_{-\infty}^{+\infty} \frac{d x}{\left|U_{n}(x)\right|^{\alpha}}=C_{1}+C_{2}
$$

where

$$
C_{1}=2 \int_{1}^{+\infty} \frac{d x}{U_{n}(x)^{\alpha}}, C_{2}=2 \int_{0}^{1} \frac{d x}{\left|U_{n}(x)\right|^{\alpha}} .
$$

We will now derive upper and lower bounds on $C_{1}$ and $C_{2}$. 
Put $C_{1}=2 \int_{1}^{+\infty} \frac{d x}{U_{n}(x)^{\alpha}}, C_{2}=2 \int_{0}^{1} \frac{d x}{\left|U_{n}(x)\right|^{\alpha}}$, so that $\int_{-\infty}^{+\infty} \frac{d x}{\left|U_{n}(x)\right|^{\alpha}}=C_{1}+C_{2}$.

First, we determine upper and lower bounds on $C_{1}$. Recall that

$$
U_{n}(x)=\frac{\left(x+\sqrt{x^{2}-1}\right)^{n+1}-\left(x-\sqrt{x^{2}-1}\right)^{n+1}}{2 \sqrt{x^{2}-1}}
$$

for all $x$ such that $|x| \geq 1$. Thus for every $\theta \in[0, \pi / 2]$ we have

$$
\begin{aligned}
(\sin \theta)^{n} U_{n}(\csc \theta) & =\frac{(1+\cos \theta)^{n+1}-(1-\cos \theta)^{n+1}}{2 \cos \theta} \\
& =\frac{\left(2 \cos ^{2} \frac{\theta}{2}\right)^{n+1}-\left(2 \sin ^{2} \frac{\theta}{2}\right)^{n+1}}{2 \cos \theta} \\
& =2^{n} \sum_{k=0}^{n}\left(\sin ^{2} \frac{\theta}{2}\right)^{k}\left(\cos ^{2} \frac{\theta}{2}\right)^{n-k} .
\end{aligned}
$$

Since

$$
\left(\cos ^{2} \frac{\theta}{2}\right)^{n}<\sum_{k=0}^{n}\left(\sin ^{2} \frac{\theta}{2}\right)^{k}\left(\cos ^{2} \frac{\theta}{2}\right)^{n-k}<(n+1)\left(\cos ^{2} \frac{\theta}{2}\right)^{n}
$$

for every $\theta \in(0, \pi / 2]$, we find that

$$
(1+\cos \theta)^{n}<(\sin \theta)^{n} U_{n}(\csc \theta)<(n+1)(1+\cos \theta)^{n}
$$

for every $\theta \in(0, \pi / 2]$.

Using the change of variables $x=\csc \theta$, as well as the lower bound in 19 , we find that

$$
\begin{aligned}
C_{1} & =2 \int_{1}^{+\infty} \frac{d x}{U_{n}(x)^{\alpha}} \\
& =2 \int_{0}^{\pi / 2} \frac{\cos \theta d \theta}{(\sin \theta)^{2} U_{n}(\csc \theta)^{\alpha}} \\
& <2 \int_{0}^{\pi / 2} \frac{\cos \theta(\sin \theta)^{n \alpha-2} d \theta}{(1+\cos \theta)^{n \alpha}} \\
& =\left.2 \frac{(\sin \theta)^{n \alpha-1}(n \alpha \cos \theta+1)}{\left(n^{2} \alpha^{2}-1\right)(1+\cos \theta)^{n \alpha}}\right|_{0} ^{\pi / 2} \\
& =\frac{2}{n^{2} \alpha^{2}-1} .
\end{aligned}
$$

A lower bound on $C_{1}$ can be obtained analogously. As a result, we get

$$
\frac{2}{n^{2} \alpha^{2}-1}(n+1)^{-\alpha}<C_{1}<\frac{2}{n^{2} \alpha^{2}-1} .
$$


It remains to determine upper and lower bounds on $C_{2}$. We apply the substitution $x=\cos (\theta /(n+1))$ and make use of the identity

$$
U_{n}\left(\cos \left(\frac{\theta}{n+1}\right)\right)=\frac{\sin \theta}{\sin \left(\frac{\theta}{n+1}\right)}
$$

as follows:

$$
\begin{aligned}
C_{2} & =2 \int_{0}^{1} \frac{d x}{\left|U_{n}(x)\right|^{\alpha}} \\
& =\frac{2}{n+1} \int_{0}^{\pi(n+1) / 2} \sin \left(\frac{\theta}{n+1}\right)^{1+\alpha}|\sin \theta|^{-\alpha} d \theta \\
& =\frac{2}{n+1} \int_{0}^{\pi(n+1) / 2} \sin \left(\frac{\theta}{n+1}\right)^{1+\alpha} d \theta+\varepsilon_{n} \\
& =2 \int_{0}^{\pi / 2}(\sin \theta)^{1+\alpha} d \theta+\varepsilon_{n} \\
& =B\left(\frac{2+\alpha}{2}, \frac{1}{2}\right)+\varepsilon_{n},
\end{aligned}
$$

where the last equality follows from $(13)$ and

$$
\varepsilon_{n}=\frac{2}{n+1} \int_{0}^{\pi(n+1) / 2} \sin \left(\frac{\theta}{n+1}\right)^{1+\alpha}\left(|\sin \theta|^{-\alpha}-1\right) d \theta .
$$

Since $\varepsilon_{n}>0$, we obtain the lower bound $C_{2}>B\left(\frac{2+\alpha}{2}, \frac{1}{2}\right)$.

To find an upper bound on $C_{2}$, note that

$$
\begin{aligned}
\varepsilon_{n} & \leq \frac{2}{n+1} \int_{0}^{\pi(n+1) / 2}\left(|\sin \theta|^{-\alpha}-1\right) d \theta \\
& =2 \int_{0}^{\pi / 2}(\sin \theta)^{-\alpha}-\pi \\
& =B\left(\frac{1-\alpha}{2}, \frac{1}{2}\right)-\pi,
\end{aligned}
$$

where the last equality follows from 13 . We conclude that

$$
B\left(\frac{2+\alpha}{2}, \frac{1}{2}\right)<C_{2} \leq B\left(\frac{2+\alpha}{2}, \frac{1}{2}\right)+B\left(\frac{1-\alpha}{2}, \frac{1}{2}\right)-\pi .
$$




\begin{tabular}{c|c|c|c|c|c|c|c|c|c|c}
$n$ & $D_{\Psi_{n}}$ & $A_{\Psi_{n}}$ & $D_{\Pi_{n}}$ & $A_{\Pi_{n}}$ & $D_{S_{n}}$ & $A_{S_{n}}$ & $D_{T_{n}}$ & $A_{T_{n}}$ & $D_{U_{n}}$ & $A_{U_{n}}$ \\
\hline 3 & 1 & $\infty$ & $2^{2} 3$ & $\infty$ & $2^{2} 3^{3}$ & 7.28585 & $2^{4} 3^{3}$ & 5.78286 & $2^{11}$ & 4.46217 \\
4 & 1 & $\infty$ & 1 & $\infty$ & $2^{2}$ & 10.4882 & $2^{17}$ & 4.30008 & $2^{16} 5^{2}$ & 3.50332 \\
5 & 5 & $\infty$ & $2^{4} 5^{3}$ & 5.78302 & $2^{12} 5^{5}$ & 4.55444 & $2^{16} 5^{5}$ & 3.78568 & $2^{28} 3^{3}$ & 3.19719 \\
6 & 1 & $\infty$ & $2^{2} 3$ & $\infty$ & $2^{16} 3^{6}$ & 5.29992 & $2^{31} 3^{6}$ & 3.52082 & $2^{36} 7^{4}$ & 3.04985 \\
7 & $7^{2}$ & 8.31171 & $2^{6} 7^{5}$ & 5.38644 & $2^{30} 7^{7}$ & 3.99650 & $2^{36} 7^{7}$ & 3.35841 & $2^{64}$ & 2.96434 \\
8 & $2^{3}$ & $\infty$ & $2^{3}$ & $\infty$ & $2^{24}$ & 6.48467 & $2^{73}$ & 3.24832 & $2^{64} 3^{12}$ & 2.90894 \\
9 & $3^{4}$ & 7.64379 & $2^{6} 3^{9}$ & 5.63543 & $2^{56} 3^{18}$ & 3.75495 & $2^{64} 3^{18}$ & 3.16867 & $2^{88} 5^{7}$ & 2.87035
\end{tabular}

Table 2: Invariants associated with $\Psi_{n}, \Pi_{n}, S_{n}, T_{n}$ and $U_{n}$ for $n=3,4, \ldots, 9$.

\section{Bean's Conjecture}

We conclude this article with the proof of Proposition 8.1, which provides further theoretical evidence in support of Conjecture 1.5 .

Proposition 8.1. For any integer $n \geq 3, Q\left(T_{n}\right) \leq Q\left(S_{n}\right)$ and $Q\left(U_{n}\right) \leq Q\left(S_{n}\right)$. Furthermore,

$$
\lim _{n \rightarrow \infty} Q\left(\Psi_{n}\right)=\lim _{n \rightarrow \infty} Q\left(\Pi_{n}\right)=\lim _{n \rightarrow \infty} Q\left(T_{n}\right)=\lim _{n \rightarrow \infty} Q\left(U_{n}\right)=\frac{16}{3} .
$$

Proof. Since $Q\left(F_{n}^{*}\right)=Q\left(S_{n}\right)$, we prove our statement with $F_{n}^{*}$ in place of $S_{n}$.

Let $n$ be an integer such that $n \geq 3$. According to Tran [14, the discriminants of $T_{n}$ and $U_{n}$ are given by

$$
D_{T_{n}}=2^{(n-1)^{2}} n^{n} \quad \text { and } \quad D_{U_{n}}=2^{n^{2}}(n+1)^{n-2} .
$$

Along with Theorems 1.3 and 1.4 , these equalities yield the upper bounds

$$
2^{-\frac{n-1}{n}} n^{-\frac{1}{n-1}} Q\left(T_{n}\right)<\frac{8}{3}+\frac{2}{3}(\sqrt[n]{4}-1)+B\left(\frac{1}{2}-\frac{1}{n}, \frac{1}{2}\right)-\pi
$$

and

$$
2^{-\frac{n}{n-1}}(n+1)^{-\frac{n-2}{n(n-1)}} Q\left(U_{n}\right)<\frac{8}{3}+\left(B\left(1+\frac{1}{n}, \frac{1}{2}\right)-2\right)+B\left(\frac{1}{2}-\frac{1}{n}, \frac{1}{2}\right)-\pi
$$

Now, the values of $Q\left(F_{n}^{*}\right)=Q\left(S_{n}\right), Q\left(T_{n}\right)$ and $Q\left(U_{n}\right)$ for $n=3,4, \ldots, 9$ are given in Table 1. The integrals associated with $A_{S_{n}}, A_{T_{n}}$ and $A_{U_{n}}$ were approximated with Mathematica. Notice that $Q\left(T_{3}\right)=Q\left(U_{3}\right)=Q\left(S_{3}\right)=3 B\left(\frac{1}{3}, \frac{1}{3}\right)$, because $S_{3}, T_{3}$ and $U_{3}$ are all equivalent under $\mathrm{GL}_{2}(\mathbb{R})$ to $x y(x-y)$. From Table 2 it is clear that the statement of Corollary 8.1 holds for the aforementioned values of $n$.

For $n \geq 10$, the inequalities $Q\left(T_{n}\right) \leq Q\left(S_{n}\right)$ and $Q\left(U_{n}\right) \leq Q\left(S_{n}\right)$ can be easily verified by combining the formula (8) with the upper bounds (21) and (22). 
The formulas for $D_{T_{n}}$ and $D_{U_{n}}$ given in 20 imply

$$
\lim _{n \rightarrow \infty} D_{T_{n}}^{1 / n(n-1)}=\lim _{n \rightarrow \infty} D_{U_{n}}^{1 / n(n-1)}=2 .
$$

Combining this with the fact that $\lim _{n \rightarrow \infty} A_{T_{n}}=\lim _{n \rightarrow \infty} A_{U_{n}}=\frac{8}{3}$, we find that

$$
\lim _{n \rightarrow \infty} Q\left(T_{n}\right)=\lim _{n \rightarrow \infty} D_{T_{n}}^{\frac{1}{n(n-1)}} A_{T_{n}}=\left(\lim _{n \rightarrow \infty} D_{T_{n}}^{\frac{1}{n(n-1)}}\right) \cdot\left(\lim _{n \rightarrow \infty} A_{T_{n}}\right)=\frac{16}{3} .
$$

The same calculation applies with $Q\left(U_{n}\right)$ in place of $Q\left(T_{n}\right)$.

Next, we prove that $\lim _{n \rightarrow \infty} Q\left(\Psi_{n}\right)=\frac{16}{3}$. For a positive integer $n$, let $\omega(n)$ denote the number of distinct prime divisors of $n$, and let $n=p_{1}^{e_{1}} \cdots p_{\omega(n)}^{e_{\omega(n)}}$ be the prime factorization of $n$. According to Liang [6], the discriminant $D_{K_{n}}$ of the number field $K_{n}=\mathbb{Q}\left(2 \cos \left(\frac{2 \pi}{n}\right)\right)$ can be computed according to the formula

$$
D_{K_{n}}= \begin{cases}2^{(m-1) 2^{m-2}-1} & \text { if } n=2^{m}, m>2, \\ p^{\left(m p^{m}-(m+1) p^{m-1}-1\right) / 2} & \text { if } n=p^{m} \text { or } 2 p^{m}, p>2 \text { prime } \\ \left(\prod_{i=1}^{\omega(n)} p_{i}^{e_{i}-1 /\left(p_{i}-1\right)}\right)^{\frac{\varphi(n)}{2}} & \text { if } \omega(n)>1, n \neq 2 p^{m} .\end{cases}
$$

It was also established by Liang that the ring of integers $\mathcal{O}_{K_{n}}$ of $K_{n}$ has a power integral basis, i.e., $\mathcal{O}_{K_{n}}=\mathbb{Z}\left[2 \cos \left(\frac{2 \pi}{n}\right)\right]$. Consequently, the discriminant $D_{\Psi_{n}}$ of $\Psi_{n}$ is equal to $D_{K_{n}}$. Since $1 \leq D_{\Psi_{n}} \leq n^{\varphi(n) / 2}$, it follows from 15 and Theorem 1.1 that

$$
\lim _{n \rightarrow \infty} Q\left(\Psi_{n}\right)=\frac{16}{3} .
$$

In view of Corollary 1.2 , this result also implies $\lim _{n \rightarrow \infty} Q\left(\Pi_{n}\right)=\lim _{n \rightarrow \infty} Q\left(\Psi_{n}\right)=\frac{16}{3}$.

\section{Acknowledgements}

The author is grateful to Prof. Cameron L. Stewart for his numerous suggestions, to Patrick Naylor for many productive conversations, and to the anonymous reviewer for their valuable recommendations.

\section{References}

[1] M. A. Bean, An isoperimetric inequality for the area of plane regions defined by binary forms, Compositio Math. 92, pp. 115-131, 1994.

[2] M. A. Bean, Binary forms, hypergeometric functions and the SchwarzChristoffel mapping formula, Trans. Amer. Math. Soc. 347, pp. 4959-4983, 1995. 
[3] M. A. Bean and R.S. Laugesen, Binary forms, equiangular polygons and harmonic measure, Rocky Mt. J. Math. 30, pp. 15-62, 2000.

[4] E. Fouvry and M. Waldschmidt, Sur la représentation des entiers par les formes cyclotomiques de grand degré, Bulletin de la SMF 148, pp. 253-282, 2020 .

[5] D. H. Lehmer, A note on trigonometric algebraic numbers, Amer. Math. Monthly 40 (3), pp. 165-166, 1933.

[6] J. J. Liang, On the integral basis of the maximal real subfield of a cyclotomic field, Journal für die reine und angewandte Mathematik 1976 (286-287), pp. 223-226, 1976.

[7] K. Mahler, Zur Approximation algebraischer Zahlen III, Acta Math. 62, pp. 91-166, 1933.

[8] J. C. Mason, D. C. Handscomb, Chebyshev Polynomials. Chapman \& Hall/CRC, 2003.

[9] A. Mosunov, On the area bounded by the curve $\prod_{1}^{n}\left|x \sin \left(\frac{k \pi}{n}\right)-y \cos \left(\frac{k \pi}{n}\right)\right|=1$, Rocky Mt. J. Math. 50 (5), pp. $k=1$
$1773-1777,2020$.

[10] J. L. Nicolas and G. Robin, Majoritations explicites pour le nombre de diviseurs de n, Bull. Can. Math. Soc. 26, pp. 485-492, 1983.

[11] I. Niven, Irrational Numbers. The Mathematical Association of America, New Jersey, 1956.

[12] J. B. Rosser and L. Schoenfeld, Approximate formulas for some functions of prime numbers, Illinois J. Math 6 (1), pp. 64-94, 1962.

[13] C. L. Stewart and S. Y. Xiao, On the representation of integers by binary forms, Math. Ann. 375, pp. 133-163, 2019.

[14] K. Tran, Discriminants of polynomials related to Chebyshev polynomials: the "Mutt and Jeff" syndrome, J. Math. Anal. Appl. 383, pp. 120-129, 2011.

[15] W. Watkins and J. Zeitlin, The minimal polynomial of $\cos (2 \pi / n)$, Amer. Math. Monthly 100 (5), pp. 471-474, 1993. 